Check for updates

Cite this: RSC Adv., 2018, 8, 41003

\title{
High removal of thiophene from model gasoline by porous MIL-101(Cr)/SA hybrid membrane $\uparrow$
}

\begin{abstract}
Wen Bing Wu, (D)*ab Sun Xue, ${ }^{a}$ Jian Hua Chen (D) ${ }^{b}$ and Xiao Liª
Membrane separation technologies have great promising potential for applications in several industries. Metal-organic frameworks (MOFs), for their large surface areas, low framework densities, transitionmetal ions in the skeleton and high pore volumes relative to other porous matrices, have great potential for the removal of sulfur from gasoline with high efficiency. In the present study, a novel porous membrane adsorbent MIL-101(Cr)/SA was prepared by immobilizing MIL-101(Cr) onto sodium alginate

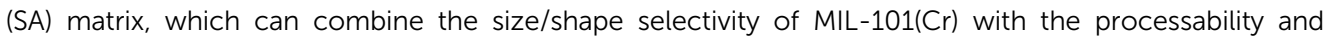
mechanical stability of SA polymer. The physico-chemical properties of MIL-101(Cr)/SA were investigated by FT-IR, SEM, BET, XRD and EDX methods. To investigate the effects of some important factors on the adsorption behavior for thiophene, a batch of experiments were performed by changing the concentration of porogen polyethylene glycol in the MIL-101(Cr)/SA, solution temperature, initial thiophene concentration and contact time. Meanwhile, benzothiophene, thiophene and 3-methyl thiophene were used to test the selectivity of MIL-101(Cr)/SA. The MIL-101(Cr)/SA showed an excellent uptake capacity of $671 \mathrm{mg} \mathrm{g}^{-1}$ under the optimal adsorption conditions. Selectivity testing indicated that the uptake capacity of MIL-101(Cr)/SA follows the order of benzothiophene > thiophene > 3-methyl thiophene. Kinetics experiments indicated the pseudo-second-order model displayed good correlation with adsorption kinetics data. The Crank model showed that the intraparticle solute diffusion is the ratecontrolling adsorption step. Regeneration experiment result shows that the prepared MIL-101(Cr)/SA has excellent adsorption and desorption efficiencies.
\end{abstract}

Received 5th August 2018

Accepted 7th November 2018

DOI: $10.1039 / c 8 r a 06579 a$

rsc.li/rsc-advances technologies can mainly be divided into hydrodesulfurization and non-hydrodesulfurization. Conventional hydrodesulfurization is highly effective for the removal of thiol, sulfides and disulfides, but less effective for thiophene and thiophene derivatives. ${ }^{5}$ Meanwhile, hydrodesulfurization needs severe conditions (up to $400{ }^{\circ} \mathrm{C}$ and $100 \mathrm{~atm}$ ), ${ }^{6}$ and what's more, hydrodesulfurization has accompanied a significant loss in octane number. ${ }^{78}$ Non-hydrodesulfurization is regarded as the future direction for gasoline desulfurization, because of its low operating cost, relatively simple technology, and mild reaction conditions. Non-hydrodesulfurization technology includes oxidation desulfurization, ${ }^{9-12}$ biological desulfurization ${ }^{13,14}$ and adsorption desulfurization, ${ }^{5,6}$ etc. Among these methods, selectively removing sulfur compounds from fuels by adsorption has received much attention because this technology shows some advantages, such as operating at ambient conditions, without compromising the quality of fuel. Meanwhile, adsorption is also able to reduce the final cost of operation and unchanging oil performance. ${ }^{15,16}$

Many studies have recently focused on novel thiophene adsorption materials including zeolites, ${ }^{17-20}$ mesoporous materials, ${ }^{21,22}$ biomaterial, ${ }^{23}$ clays, ${ }^{24}$ metal oxides, ${ }^{25}$ activated alumina, ${ }^{26}$ etc., However, adsorption desulfurization process still has some remaining issues, such as, a limited saturated
${ }^{a}$ School of Chemical Engineering, Fuzhou University, Fuzhou 350108, China. E-mail: wbwu81@126.com; lxzwy@fzu.edu.cn; Fax: +86-596-2520035; +86-591-87892513; Tel: +86-596-2591445; +86-591-87892513

${ }^{b}$ College of Chemistry, Chemical Engineering and Environment, Minnan Normal University, Zhangzhou 363000, China

$\dagger$ Electronic supplementary information (ESI) available. See DOI: 10.1039/c8ra06579a 
adsorption capacity, low selectivity, unsatisfactory adsorption rate or regeneration of the adsorbents, which make the adsorption process difficult to put into practice in large scale applications. Thus, developing some novel adsorbents that simultaneously possesses expected adsorption capacity and high selectivity for thiophene, and also easy for regeneration has been an interesting research topic. Many of researches indicate that the transition-metal ions play an essential function in adsorptive desulfurization process. ${ }^{19,20,22,27}$ In the adsorption process, the metal cations can form the usual $\sigma$ bonds with pi-electrons of adsorbates (thiophene), in addition, their d-orbitals can back-donate electron density to the antibonding p-orbitals of the sulfur ring to form back donating pibond. The adsorption occurs due to the $\sigma$-pi bonds synergistic effect between metal ions and adsorbates. ${ }^{28}$ Recently, metal organic frameworks (MOFs) have been extensively explored. MOFs are composed of metal ions or metal oxide clusters and organic bridging ligands to construct variety of frameworks by selecting and combining building units. ${ }^{29}$ As a result, they have obtained great attention due to their large surface areas, low framework densities, and high pore volumes relative to other porous matrices. ${ }^{30}$ They have significant potential for use in a variety of applications, including storage of gases, selective gas separations and especially for adsorptive desulfurization. .1,32 $^{31}$

Recently, owing to the faster diffusion rate of the adsorbates into the internal active sites in the adsorptive porous membrane than that of traditional porous adsorptive materials, adsorptive porous membrane is now gaining more and more attention. ${ }^{33-35}$ However, few researches have been done on the adsorption properties of the porous membranes on the desulfurization. Sodium alginate (SA) is a kind of abundant, biodegradable, nontoxic biopolymers. For its good membrane forming property, high activity of carbonyl groups and hydroxyl groups on its chains which are excellent functional groups for anchoring adsorbates, SA has attracted considerable attention currently. ${ }^{36}$ In the present paper, we synthesized metal-organic frameworks (MOFs) MIL-101(Cr). The prepared MIL-101(Cr) has large specific surface area of $4315 \mathrm{~m}^{2} \mathrm{~g}^{-1}$, good thermal stability, $\pi-\pi$ interactions/stacking, hydrogen bonding forming property, and pore/size-selective adsorption property. Based on above, MIL$101(\mathrm{Cr})$ appear to be act as promising sulfur adsorbents. Thus, MIL-101(Cr) acted as an adsorptive material was immobilized onto sodium alginate (SA) matrix and using polyvinylpyrrolidone as a porogen to prepare a novel porous hybrid membrane adsorbent MIL-101(Cr)/SA. Physico-chemical properties of the MIL-101(Cr)/SA were investigated by SEM, FT-IR, BET, XRD and AFM methods. The porous MIL-101(Cr)/SA hybrid membrane was used for desulfurization of model gasoline containing thiophene. Batch adsorption experiments were carried out to investigate the effects of process factors, such as the concentration of porogen polyethylene glycol in the porous MIL-101(Cr)/SA hybrid membrane, solution temperature, initial thiophene concentration and contact time on adsorption property. Meanwhile, the selectivity and regeneration of porous MIL-101(Cr)/SA hybrid membrane were also investigated. The adsorption experiment results indicated that the prepared porous MIL-101(Cr)/SA hybrid membrane exhibited excellent desulfurization ability.

\section{Experimental section}

\subsection{Materials}

Chromium(III) nitrate nonahydrate $\left(\mathrm{Cr}\left(\mathrm{NO}_{3}\right)_{3} \cdot 9 \mathrm{H}_{2} \mathrm{O}, \mathrm{AR}\right)$, sodium hydroxide ( $\mathrm{NaOH}, \mathrm{AR})$, sodium acetate ( $\mathrm{NaAc}, \mathrm{AR}$ ), sodium alginate (SA, CP), and polyvinylpyrrolidone (AR) were purchased from West Long chemical. Terephthalic acid $\left(\mathrm{H}_{2} \mathrm{BDC}, \mathrm{AR}\right)$, thiophene (AR), benzothiophene (AR), and 3methylthiophene (AR) were bought from Aladdin Corp (Shanghai, China). Hydrochloric acid ( $\mathrm{HCl}, 99 \%)$, ethanol (AR) were from Medicines reagents. Deionized water was prepared by our laboratory.

\subsection{Preparation porous MIL-101(Cr)/SA hybrid membrane}

2.2.1 Synthesis of MIL-101(Cr). The preparation of MIL$101(\mathrm{Cr})$ nanoparticles was derived from literature. ${ }^{37}$ Briefly, $4.0 \mathrm{~g}$ (0.01 mol) chromic(III) nitrate nonahydrate and $1.64 \mathrm{~g}(0.01$ mol) terephthalic acid were added into $50 \mathrm{~mL} 0.05 \mathrm{~mol} \mathrm{~L}^{-1} \mathrm{SA}$ solution under stirring, and then a dark-green turbid mixture was obtained. The mixture was put into a $125 \mathrm{~mL}$ stainless steel autoclave with a polytetrafluoroethylene liner. The autoclave was heated from room temperature to $170{ }^{\circ} \mathrm{C}$ within $40 \mathrm{~min}$, and then held for $24 \mathrm{~h}$. After natural cooling for $12 \mathrm{~h}$, the MIL$101(\mathrm{Cr})$ nanoparticles were separated from the mixture by filtration with $0.4 \mu \mathrm{m}$ membrane. In order to purify MIL-101(Cr) nanoparticles, it was dispersed in $100 \mathrm{~mL}$ ethanol and sonicated for $30 \mathrm{~min}$ to remove the terephthalic acid, then followed by centrifugation. Finally, the MIL-101(Cr) nanoparticles product was dried under vacuum at $100{ }^{\circ} \mathrm{C}$ over the night.

2.2.2 Preparation of porous MIL-101(Cr)/SA hybrid membrane. SA $(1.0 \mathrm{~g})$ and Polyvinylpyrrolidone $(0.3 \mathrm{~g})$ were dissolved in $40 \mathrm{~mL}$ of deionized water by constant stirring at room temperature. Meanwhile, a certain amount of MIL-101(Cr) nanoparticles were dispersed in $40 \mathrm{~mL}$ of deionized water and sonicated for $10 \mathrm{~min}$. Then, the well dispersed MIL-101(Cr) nanoparticles were added into the previously prepared SA solution. Finally, the solution was further stirred for $12 \mathrm{~h}$ and kept overnight to release any gases. The obtained homogeneous solution was poured onto a clean glass plate and heated in an oven at $60{ }^{\circ} \mathrm{C}$ for $2 \mathrm{~h}$. Then the dried porous MIL-101(Cr)/SA hybrid membrane was gently peeled off from the glass plate and soaked in anhydrous ethanol for $24 \mathrm{~h}$ to wash out porogen. Then the porous MIL-101(Cr)/SA hybrid membrane was further heated in an oven at $60{ }^{\circ} \mathrm{C}$ for $30 \mathrm{~min}$.

\subsection{Characterization of porous MIL-101(Cr)/SA hybrid membrane}

The porous MIL-101(Cr)/SA hybrid membrane were observed by XRD (Bruker D8 Advance with $\mathrm{Cu} / \alpha$ radiation), FT-IR (Nicolet 8700), field emission scanning electron microscopy (SEM, HITACHI S-4800) and Energy Dispersion Spectrum (EDS). The morphologies of porous MIL-101(Cr)/SA hybrid membrane before and after adsorption were also studied by Atomic Force 
Microscope (AFM, CSPM5500). The thiophene in the $N$-heptane solutions was tested by Gas Chromatograph (GC, G5).

\subsection{Adsorption experiments}

The model gasoline were prepared by dissolved various amount of thiophene in $N$-heptane. For each adsorption equilibration experiment, $0.025 \mathrm{~g}$ of the adsorbent was added to $25.0 \mathrm{~mL}$ of solution (e.g., thiophene concentrations of 100, 300, 500, 700 and $900 \mathrm{mg} \mathrm{L}^{-1}$ ) and shaken at $150 \mathrm{rpm}$ for $24 \mathrm{~h}$ at $30{ }^{\circ} \mathrm{C}$. The effect of temperature on adsorption was determined using $1.0 \mathrm{~g}$ $\mathrm{L}^{-1}$ of adsorbent, $500 \mathrm{mg} \mathrm{L}^{-1}$ thiophene solution at different temperatures $\left(25,30,35,40\right.$ and $\left.45^{\circ} \mathrm{C}\right)$. Adsorption kinetics was carried out by withdrawing $5.0 \mathrm{~mL}$ of sample from solution at regular intervals. The concentration of thiophene in the solution was determined by gas chromatograph.

\subsection{Desorption and repeatability test}

$0.025 \mathrm{~g}$ of porous MIL-101(Cr)/SA hybrid membrane was added to $25 \mathrm{~mL}$ of $500 \mathrm{mg} \mathrm{L}^{-1}$ thiophene solution shaken at $150 \mathrm{rpm}$ for $24 \mathrm{~h}$ at $30{ }^{\circ} \mathrm{C}$. Then the adsorbed porous MIL-101(Cr)/SA hybrid membrane was dipped into $100 \mathrm{~mL}$ of anhydrous ethanol to removed thiophene. Repeat the adsorption-desorption tests to investigate the repeatability of porous MIL-101(Cr)/ SA hybrid membrane.

\subsection{The selective of porous MIL-101(Cr)/SA hybrid membrane}

The concentration of thiophene, 3-methyl thiophene and benzothiophene were analysed by gas ghromatograph. The amount of the adsorbed sulfide onto porous MIL-101(Cr)/SA hybrid membrane was calculated using the following equation:

$$
q_{\mathrm{e}}=\frac{\left(C_{0}-C_{\mathrm{e}}\right) \times V}{m}
$$

where $q_{\mathrm{e}}$ is adsorbed amount on the adsorbents $\left(\mathrm{mg} \mathrm{g}^{-1}\right) ; C_{0}$ and $C_{\mathrm{e}}$ are the initial and equilibrium concentration of the adsorbate $\left(\mathrm{mg} \mathrm{L}^{-1}\right)$, respectively; $V$ is the solution volume $(\mathrm{mL})$ and $m$ is the adsorbents dosage (g).

\subsection{Effect of toluene on adsorption property}

Generally, there are some aromatic hydrocarbons in gasoline, which can affect the adsorption property on porous MIL101(Cr)/SA hybrid membrane for thiophene. For each adsorption equilibration experiment, $0.025 \mathrm{~g}$ of the adsorbent was added to $25.0 \mathrm{~mL}$ of solution (e.g., thiophene and toluene concentrations of 100, 300, 500, 700 and $900 \mathrm{mg} \mathrm{L}^{-1}$ ) and shaken at $150 \mathrm{rpm}$ for $24 \mathrm{~h}$ at $30^{\circ} \mathrm{C}$.

\section{Results and discussion}

\subsection{Characterization of membranes}

The surface morphology of porous MIL-101(Cr)/SA hybrid membrane before and after adsorption and element distribution mapping of $\mathrm{S}$ after adsorption of thiophene was observed by SEM, as shown in Fig. 1(a) and (b). It indicates that porous MIL-101(Cr)/SA hybrid membrane present a three dimensional porous structure, and thiophene was homogenously adsorbed on the porous MIL-101(Cr)/SA hybrid membrane. The porous MIL-101(Cr)/SA hybrid membranes before and after adsorption of thiophene were also investigated by FT-IR, as shown in Fig. 1(c). The band at $3400-3000 \mathrm{~cm}^{-1}$ was ascribed to the stretching vibration of $-\mathrm{OH}$; the band at $3100-3000 \mathrm{~cm}^{-1}$ showed the stretching vibration of $\mathrm{C}-\mathrm{H}$ bond on the benzene ring; the band at $2800 \mathrm{~cm}^{-1}$ showed the dissymmetric stretching vibration of $-\mathrm{CH}_{2}$ and symmetric stretching vibration of $-\mathrm{CH}$, respectively; the band at $1770-1680 \mathrm{~cm}^{-1}$ revealed the stretching vibration of $-\mathrm{C}=\mathrm{O}$; the band at $1600 \mathrm{~cm}^{-1}$ and $1450 \mathrm{~cm}^{-1}$ were attributed to stretching vibration of benzene ring skeleton; the band at $900-650 \mathrm{~cm}^{-1}$ showed the out-ofplane bending vibration of $\mathrm{C}-\mathrm{H}$ on the benzene ring. ${ }^{38}$ From Fig. 1(c), one can also find that after adsorption of thiophene, duo to $\pi$-complexation and direct coordination $(\mathrm{S}-\mathrm{M})$ interaction between porous adsorbent and thiophene, some of characteristic band, such as stretching vibration of benzene ring skeleton, have slightly shifted from $1513.16 \mathrm{~cm}^{-1}$ to a high wave number of $1528.35 \mathrm{~cm}^{-1} .{ }^{39}$

The BET surface area of the prepared porous MIL-101(Cr)/SA hybrid membrane was obtained on the basis of nitrogen adsorption-desorption isotherms at $-196{ }^{\circ} \mathrm{C}$ and shown in Fig. 1(d). It can be found that the adsorption isotherm of porous MIL-101(Cr)/SA hybrid membrane is typical of Type IV with a hysteresis loop. Therefore, there are mesoporous structures in the material. Surface area of the porous MIL-101(Cr)/SA hybrid membrane calculated form BET analysis was $5.56 \mathrm{~m}^{2} \mathrm{~g}^{-1}$, which was significant higher than that of pristine SA membrane $(0.37$ $\mathrm{m}^{2} \mathrm{~g}^{-1}$ ). But with the progress of adsorption experiments, the BET surface area of the porous MIL-101(Cr)/SA hybrid membrane was $5.11 \mathrm{~m}^{2} \mathrm{~g}^{-1}$, which was $0.45 \mathrm{~m}^{2} \mathrm{~g}^{-1}$ decreased.

AFM was also used to assess the changes appeared in surface morphology and roughness of the prepared porous MIL101(Cr)/SA hybrid membranes. In Fig. 1(e) and (f) depicts the AFM images of pristine SA membrane and porous MIL-101(Cr)/ SA hybrid membrane. A variation in surface morphology and an enhancing in surface roughness are observed distinctly when the MIL-101(Cr) crystal nanoparticles were incorporated into SA matrix. An increasing of roughness of the porous MIL-101(Cr)/ SA hybrid membranes will increase the specific surface area and active sites of porous MIL-101(Cr)/SA hybrid membrane. As a result, adsorption capacity of porous MIL-101(Cr)/SA hybrid membrane for thiophene will be improved.

\subsection{Adsorption test}

In liquid phase adsorption, the adsorption capacity of adsorbent for adsorbate depends on a number of factors such as the physical properties (pore size, heat stability, size distribution, et al.), chemical properties (functional group, the polarity of the material, et al.), and environmental factor (temperature, concentration, time, toluene, et al.). Therefore, the effects of several factors on the adsorption performance were carried out.

3.2.1 The effect of the amount of PEG on adsorption property. To investigate the effect of the amount of porogen PEG on adsorption property, adsorption experiments were 

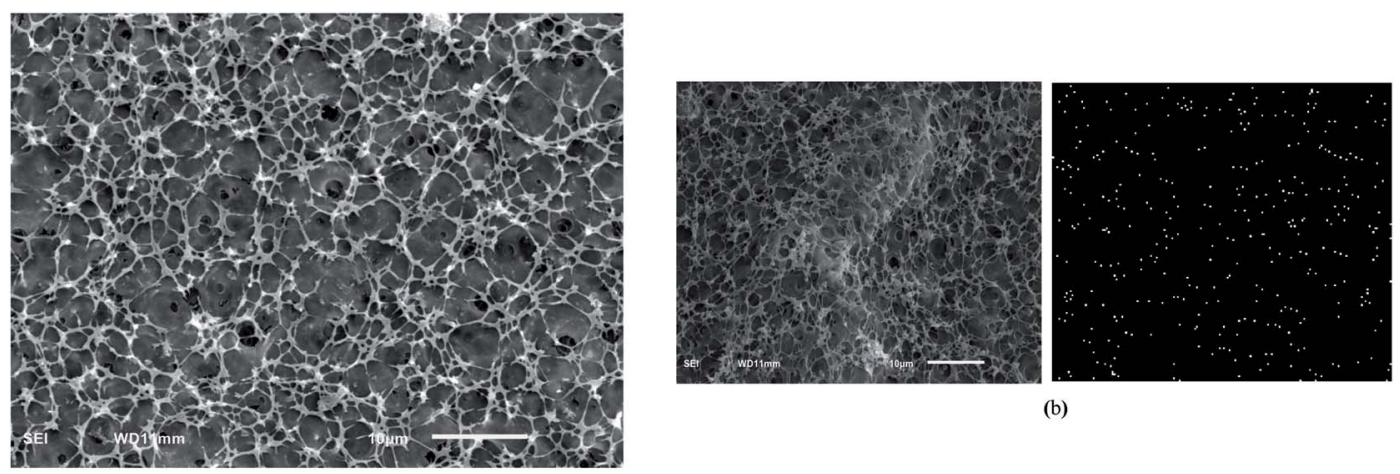

(b)

(a)

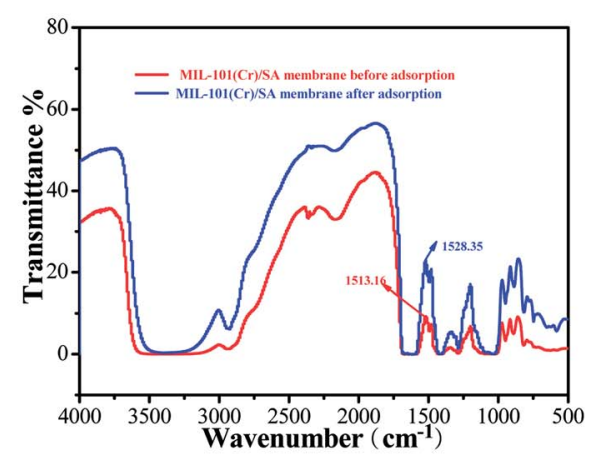

(c)

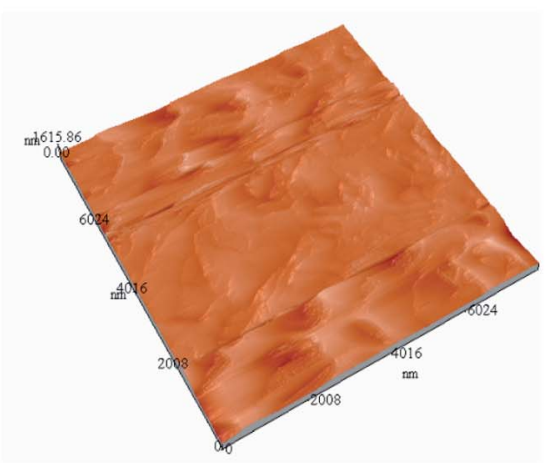

(e)

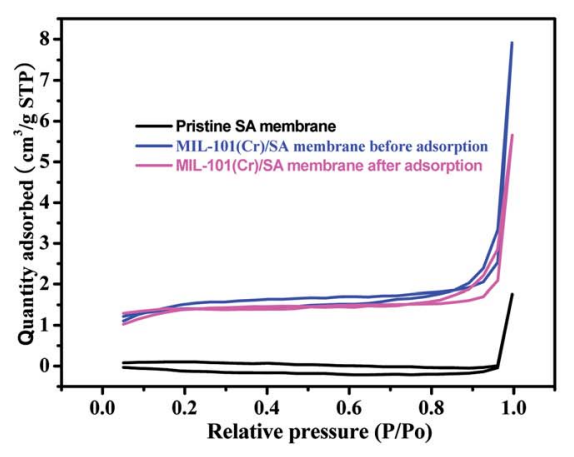

(d)

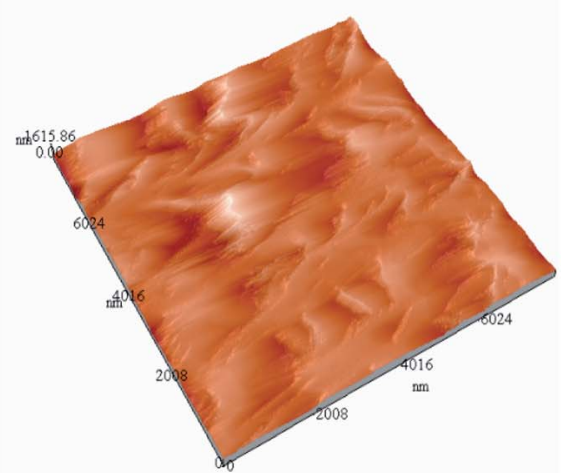

(f)

Fig. 1 SEM image of (a) the porous MIL-101(Cr)/SA hybrid membrane before adsorption, (b) elemental distribution mapping of S after adsorption of thiophene, (c) FT-IR spectra of porous MIL-101(Cr)/SA hybrid membrane before and after adsorption, (d) $\mathrm{N}_{2}$ adsorption analysis the BET surface area of pristine SA membrane, and porous MIL-101(Cr)/SA hybrid membrane before and after adsorption. (e) AFM image of pristine membrane, and (f) AFM image of the porous MIL-101(Cr)/SA hybrid membrane.

performed and shown in Fig. 2. It can be observed that the saturated adsorption capacity of the porous MIL-101(Cr)/SA hybrid membrane for thiophene obtains the maximum $671 \mathrm{mg} \mathrm{g}^{-1}$ at the amount of PEG of $23 \mathrm{wt} \%$. This may be attributed to the specific surface of the porous MIL-101(Cr)/SA hybrid membrane raised with an increase of PEG amount, which provided more adsorption sites for adsorption thiophene. When the amount of PEG higher than $23 \mathrm{wt} \%$, due to the aggregation of the PEG in the MIL-101(Cr)/SA matrix, the specific surface of the porous MIL-101(Cr)/SA hybrid membrane decreased, hence the saturated adsorption capacity of the porousMIL-101(Cr)/SA hybrid membrane also decreased. Therefore, the porous MIL-101(Cr)/SA hybrid membrane with PEG amount of $23 \mathrm{wt} \%$ was used for further adsorption experiments.

3.2.2 The effect of adsorbate concentration on adsorption property. The concentration of adsorbate plays an important role in the adsorption process. Fig. 3 indicated that the adsorption capacity of the porous MIL-101(Cr)/SA hybrid membranes increased on account of the increased 


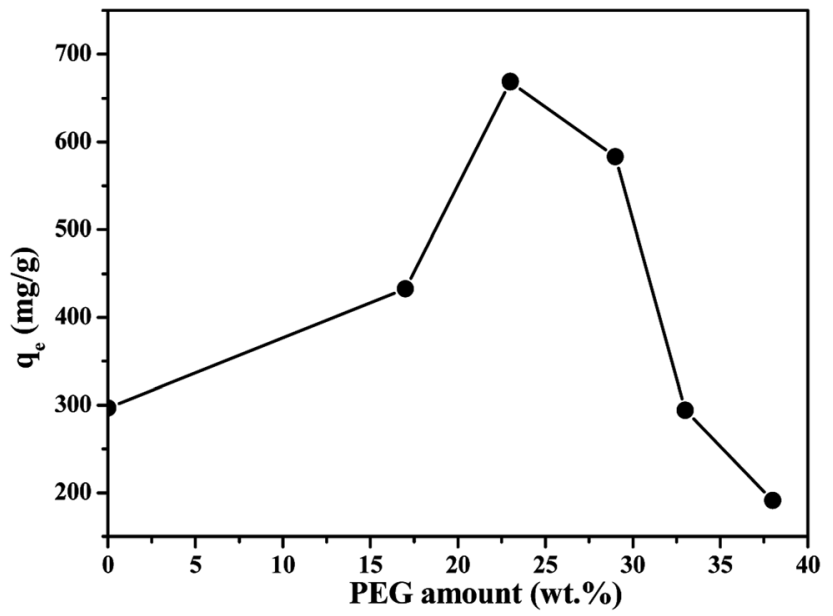

Fig. 2 The effect of the amount of porogen PEG on adsorption property: thiophene initial concentration $500 \mathrm{mg} \mathrm{L}^{-1}$, adsorbent dosage $0.25 \mathrm{~g} \mathrm{~L}^{-1}$, stirring speed $150 \mathrm{rpm}$, contact time $24 \mathrm{~h}$.

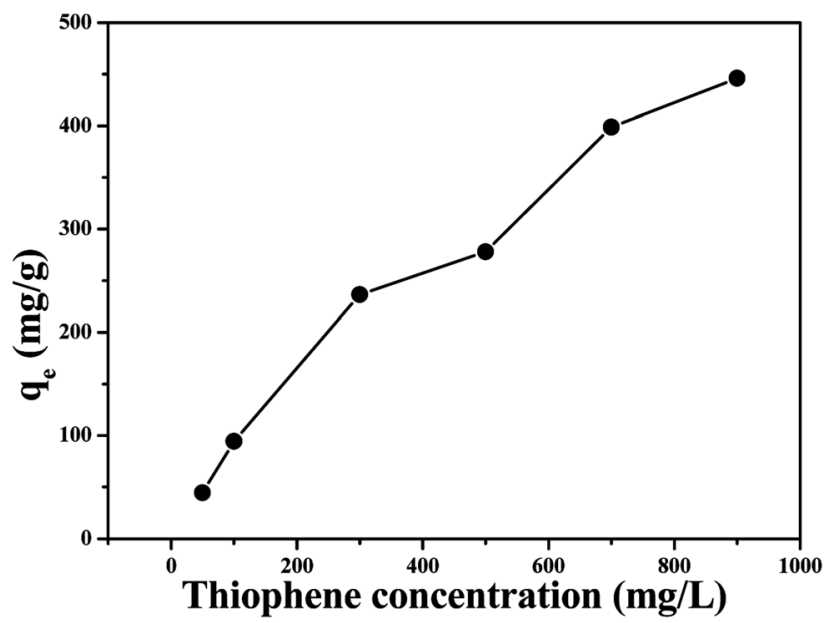

Fig. 3 The effect of adsorbate concentration on adsorption property: adsorbent dosage $1.0 \mathrm{~g} \mathrm{~L}^{-1}$, temperature $308 \mathrm{~K}$, stirring speed $150 \mathrm{rpm}$, contact time $24 \mathrm{~h}$.

concentration of the thiophene. Because the adsorptiondesorption process is a reversible equilibrium process. With the increase of adsorbate concentration, it can provide stronger driving force to improve the adsorption capacity. Secondly, an increase of initial thiophene concentration may also lead to a stronger interaction between the thiophene and MIL-101(Cr)/ SA hybrid membranes.

3.2.3 The effect of temperature on adsorption property. It has been recognized that the adsorption of adsorbate from a solution by adsorbent is affected by the temperature. An increasing in temperature is known to increase the diffusion rate of the adsorbate molecules greatly. Furthermore, changing the temperature will lead to a new equilibrium capacity of the adsorbent for a particular adsorbate. The effect of temperature on the adsorption capacities of the porous MIL-101(Cr)/SA hybrid membranes towards thiophene was presented in

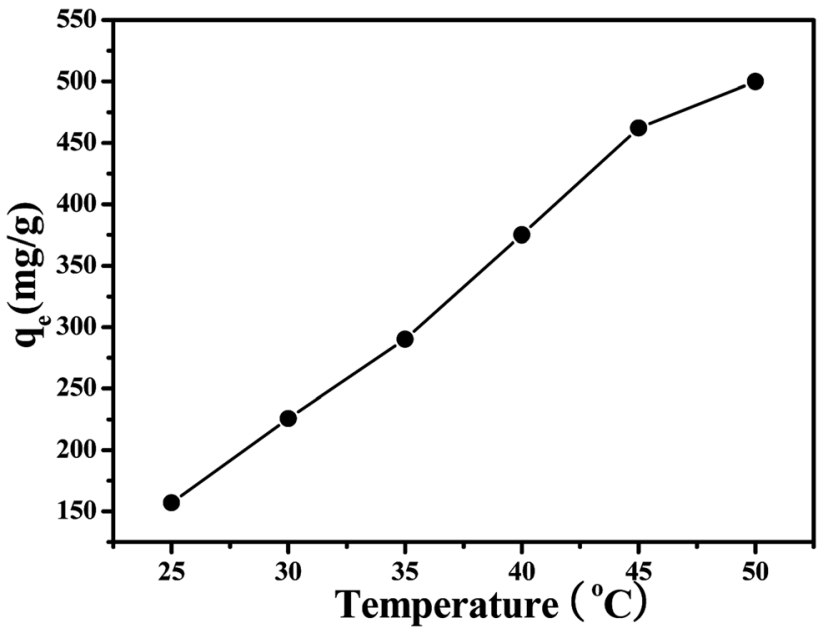

Fig. 4 Effect of temperature on adsorption property: thiophene initial concentration $500 \mathrm{mg} \mathrm{L}^{-1}$, adsorbent dosage $1.0 \mathrm{~g} \mathrm{~L}^{-1}$, stirring speed $150 \mathrm{rpm}$, contact time $24 \mathrm{~h}$.

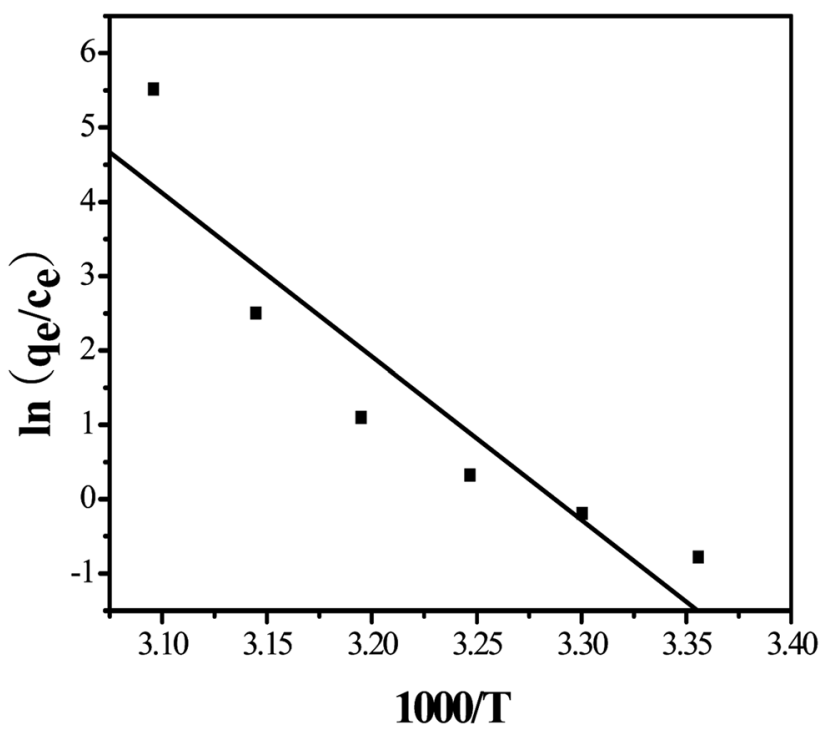

Fig. 5 Linear plot of In $K^{0} v s .1000 / T$ for the adsorption of thiophene on the hybrid membrane.

Fig. 4. It showed that the adsorption capacity increased slowly with an increasing of temperature. This indicated an endothermic nature of the adsorption process.

The thermodynamic model and the thermodynamic parameters $\left(\Delta H^{0}, \Delta S^{0}\right.$, and $\left.\Delta G^{0}\right)$ can be calculated using the following equations:

$$
\begin{gathered}
\ln K^{0}=-\Delta H^{0} / R T+\Delta S^{0} / R \\
\Delta G^{0}=-R T \ln K^{0} \\
K^{0}=\left(C_{0}-C_{\mathrm{e}}\right) V / m C_{\mathrm{e}}
\end{gathered}
$$

where, $R$ is the universal gas constant $\left(8.314 \mathrm{~J} \mathrm{~mol}^{-1} \mathrm{~K}^{-1}\right)$ and $T$ is the absolute temperature (in kelvin). Plotting $\ln K^{0}$ against 
Table 1 Parameters obtained from different kinetic models

\begin{tabular}{|c|c|c|c|c|}
\hline Adsorbent & $T / \mathrm{K}$ & $\Delta G\left(\mathrm{~kJ} \mathrm{~mol}^{-1}\right)$ & $\Delta H\left(\mathrm{~kJ} \mathrm{~mol}^{-1}\right)$ & $\Delta S\left(\mathrm{~J} \mathrm{~mol}^{-1} \mathrm{~K}^{-1}\right)$ \\
\hline \multirow[t]{5}{*}{ MIL-101(Cr)/SA hybrid membrane } & 298 & 4.256 & 183.5 & 601.487 \\
\hline & 303 & 1.249 & & \\
\hline & 313 & -4.765 & & \\
\hline & 318 & -7.772 & & \\
\hline & 323 & -10.780 & & \\
\hline
\end{tabular}

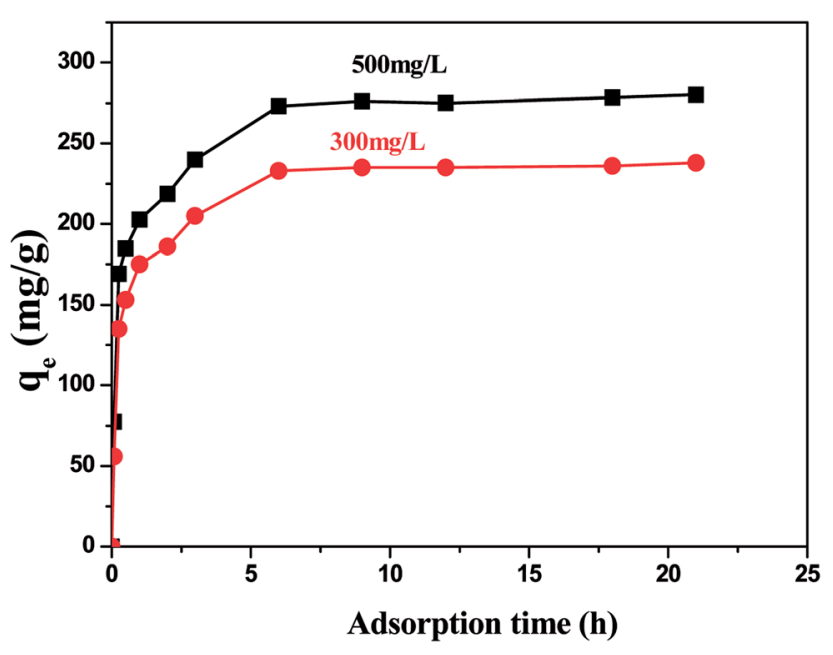

Fig. 6 Effect of reaction time on adsorption property: thiophene initial concentration $100 \mathrm{mg} \mathrm{L}^{-1}$, adsorbent dosage $0.2 \mathrm{~g} \mathrm{~L}^{-1}$, stirring speed $150 \mathrm{rpm}$, contact time $24 \mathrm{~h}$.

$1000 / T$ gives a straight line, as shown in Fig. 5, with the slope equal to $-\Delta H^{0} / R$. Thermodynamic parameters were calculated according to eqn (2)-(4). As shown in Table 1 , the negative $\Delta G^{0}$ values indicates that the adsorption process is spontaneous, and the values decreases with an increase of temperature indicating a better adsorption performance is obtained at higher temperature. The positive value of $\Delta H^{0}$ confirms the endothermic nature of the overall adsorption process and positive value of $\Delta S^{0}$ suggests an increasing randomness at the solid/solution interface during the adsorption process.

3.2.4 Adsorption kinetics. The kinetics of adsorption plays an important role on the point of view that it controls the process efficiency. Adsorption dynamics of thiophene by the porous MIL-101(Cr)/SA hybrid membranes were investigated at solution concentration of $500 \mathrm{mg} \mathrm{\textrm {L } ^ { - 1 }}$, and temperature of $35^{\circ} \mathrm{C}$. As shown in Fig. 6, fast adsorption kinetics was observed. In just a few hours, we can find that adsorption capacity almost attained equilibrium.
Table 3 Kinetic data from Crank model and Biot number

\begin{tabular}{lllll}
\hline Adsorbate & $C_{0}\left(\mathrm{mg} \mathrm{L}^{-1}\right)$ & $k_{\mathrm{f}}\left(\mathrm{m} \mathrm{s}^{-1}\right)$ & $D_{\mathrm{s}}\left(\mathrm{m}^{2} \mathrm{~s}^{-1}\right)$ & Biot \\
\hline Thiophene & 500 & $1.863 \times 10^{-4}$ & $3.47 \times 10^{-11}$ & 125 \\
& 300 & $1.352 \times 10^{-4}$ & $2.38 \times 10^{-11}$ & 103
\end{tabular}

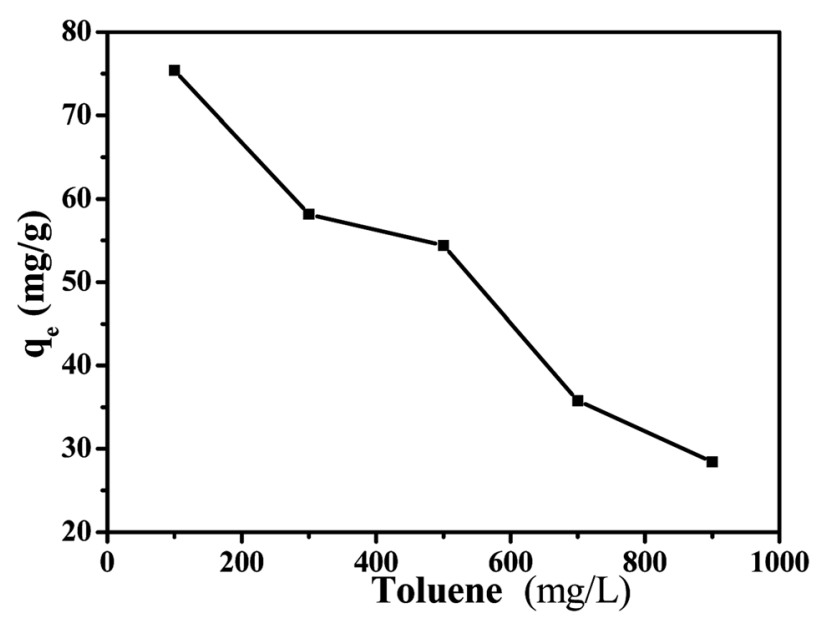

Fig. 7 Effect of toluene concentration on adsorption property: thiophene initial concentration $500 \mathrm{mg} \mathrm{L}^{-1}$, adsorbent dosage $1.0 \mathrm{~g} \mathrm{~L}^{-1}$, stirring speed $150 \mathrm{rpm}$, contact time $24 \mathrm{~h}$.

In order to examine the adsorption kinetics of the porous MIL-101(Cr)/SA hybrid membranes for thiophene, we examined:

(1) The Lagergren pseudo-second-order kinetic model (PFO)

$$
\frac{t}{q_{t}}=\left(\frac{1}{k_{2} q_{\mathrm{e}}^{2}}\right)+\frac{t}{q_{\mathrm{e}}}
$$

where $q_{\mathrm{e}}$ and $q_{t}$ denote the amounts of adsorption at equilibrium and at time $t\left(\mathrm{mg} \mathrm{g}^{-1}\right), k_{2}$ are the first order and second order rate constants $\left(\mathrm{g} \mathrm{mg}^{-1} \mathrm{~min}^{-1}\right)$, respectively.

Table 2 The Lagergren pseudo-second-order rate parameters for thiophene adsorption onto porous MIL-101(Cr)/SA hybrid membrane

\begin{tabular}{|c|c|c|c|c|c|}
\hline Adsorbate & $C_{0}\left(\mathrm{mg} \mathrm{L}^{-1}\right)$ & $q_{\mathrm{e}, \exp }\left(\mathrm{mg} \mathrm{g}^{-1}\right)$ & $k_{2}\left(\times 10^{-4} \mathrm{~g} \mathrm{mg}^{-1} \min ^{-1}\right)$ & $q_{\mathrm{e}, \mathrm{cal}}\left(\mathrm{mg} \mathrm{g}^{-1}\right)$ & $R^{2}$ \\
\hline Thiophene & 500 & 276 & 6.15 & 271 & 0.9999 \\
\hline
\end{tabular}




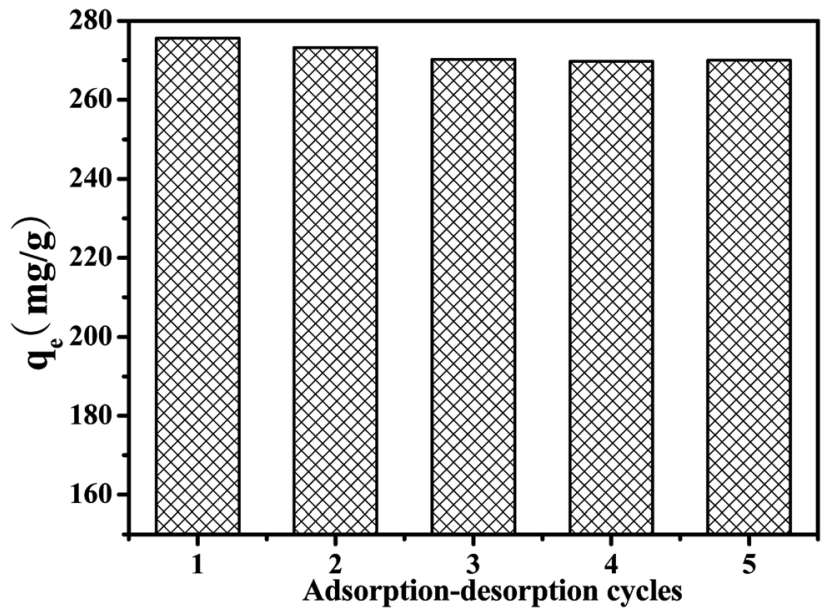

Fig. 8 Adsorption-desorption cycles.

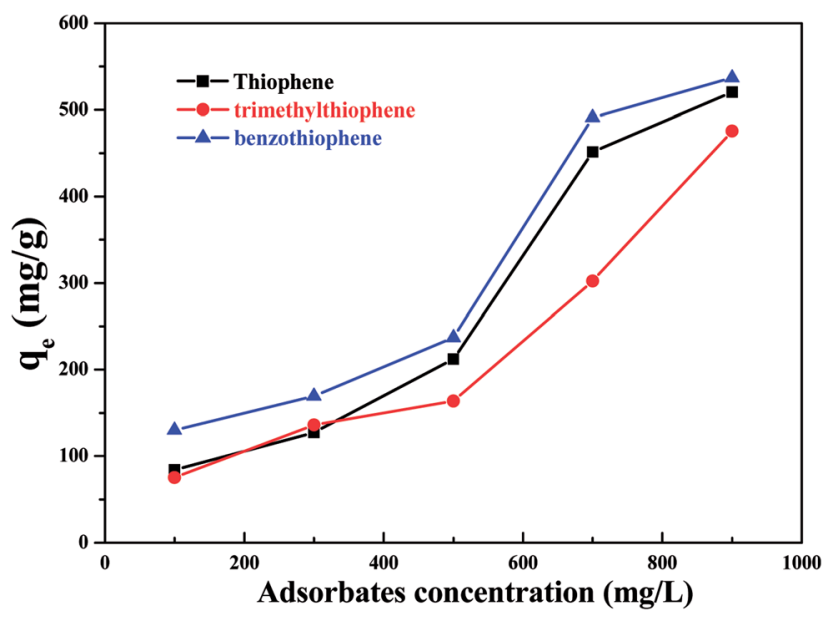

Fig. 9 Selectivity studies.

According to the kinetic formula, the experimental data were examined, and the constants that related to the model are shown in Table 2. It can be find that the pseudo-second-order equation appears to fit the model well. Meanwhile, the calculated amount of adsorption equilibrium capacity $\left(q_{\mathrm{e}, \mathrm{cal}}\right)$ from the PFO equation is close to the actual amount of adsorption equilibrium capacity ( $\left.q_{\mathrm{e}, \exp }\right)$.

(2) Crank model

The following Crank diffusion model ${ }^{40}$ has usually been used to study the adsorption kinetics data. The mass transfer rate, $N_{\mathrm{t}}$, at the external surface of the adsorbent is given by:

$$
N_{t}=\frac{\partial C_{t}}{\partial t}=k_{\mathrm{f}} S_{\mathrm{A}}\left(C_{t}-C_{\mathrm{s}}\right)
$$

where $C_{t}$ and $C_{\mathrm{s}}$ are bulk liquid phase concentration and liquid phase concentration at the surface in equilibrium with the solid phase concentration, $\mathrm{mg} \mathrm{\textrm {L } ^ { - 1 }}$, respectively; $k_{\mathrm{f}}$ is external mass transfer coefficient, $\mathrm{m} \mathrm{s}^{-1}$, and $S_{\mathrm{A}}$ is specific surface area of adsorbent, $\mathrm{m}^{2} \mathrm{~g}^{-1}$.
The following expression is obtained by integrating the eqn (6) above:

$$
\ln \left(\frac{C_{t}}{C_{0}}\right)=-k_{\mathrm{f}} S_{\mathrm{A}} t
$$

Crank also developed the following equation for slab particle: ${ }^{11}$

$$
\frac{q_{t}}{q_{\mathrm{e}}}=1-\sum_{n=1}^{\infty} \frac{6}{\pi^{2} n^{2}} \times \exp \left(\frac{-n^{2} \pi^{2} D_{\mathrm{S}} t}{r^{2}}\right)
$$

where $D_{\mathrm{S}}\left(\mathrm{m}^{2} \mathrm{~s}^{-1}\right)$ is the effective intraparticle diffusion coefficient of the adsorbate in the slab particle, $r\left(\mathrm{~m}^{-1}\right)$ is the distance from the center.

The Biot number $\left(B_{\mathrm{i}}\right.$, dimensionless) is the ratio of external transport to internal transport, ${ }^{41}$ which can be used to determine if the adsorption process is controlled by the mass transport of adsorbate in the external boundary layer of the adsorbent or by the adsorbate diffusion inside the pore of the adsorbent.

$$
B_{\mathrm{i}}=\frac{k_{\mathrm{f}} L_{\mathrm{f}}}{D_{\mathrm{S}}}
$$

where, $L_{\mathrm{f}}(\mathrm{m})$ is the thickness of the film. When $B_{\mathrm{i}} \ll 1$, the external mass transfer is the controlling step. However, when $B_{\mathrm{i}}$ $\gg 1$, the adsorbate diffusion inside the pore of the membrane become the predominant step. The $B_{\mathrm{i}}$ values calculated with experimental data are presented in Table 3. It can be find that all of $B_{\mathrm{i}}$ values are significant higher than 1, indicating that the adsorption process is mainly controlled by the intraparticle diffusion.

3.2.5 The effect of toluene concentration on adsorption property. Generally, aromatic hydrocarbons in the gasoline can affect the adsorption property of adsorbent for thiophene. Gasoline is a complex mixture of hydrocarbons composed of a certain quantity of aromatics. Therefore, we used toluene as a coexistence aromatic hydrocarbon to study the effect of aromatic hydrocarbons on the adsorption of hybrid membrane. The effects of toluene on the adsorption capacities of the porous MIL-101(Cr)/SA hybrid membranes towards thiophene were presented in Fig. 7. With the increase of the concentration of toluene, adsorption capacity showed a trend of decline indicates that toluene inhibition adsorption ability of the porous MIL-101(Cr)/SA hybrid membrane adsorption for thiophene. Transition metal ions are believed to have desulfurization property on the basis of their $\pi$-complexation type interactions with the sulfur species. Aromatics can also get adsorbed by the transition metal ions since they can also compete with thiophene through interaction of their $\pi$-electron cloud. As a result, adsorption ability of porous MIL-101(Cr)/SA hybrid membranes for thiophene became weaken.

3.2.6 Desorption studies. Regeneration was a key factor for an effective absorbent. The adsorbents with excellent regenerability can reduce the adsorbent cost greatly, which was very important for industrial applications. In this study, the solvent elution was adopted and the thiophene-loaded porous MIL$101(\mathrm{Cr}) / \mathrm{SA}$ hybrid membrane was regenerated with ethanol 
solution. The effects of the regeneration times on adsorption capacity are shown in Fig. 8. It indicates that under five times of adsorption, the as-prepared porous MIL-101(Cr)/SA hybrid membrane still remains a high adsorption capacity.

3.2.7 Selectivity studies. In order to investigate the adsorption selectivity of the porous MIL-101(Cr)/SA hybrid membrane, we selected 3-methyl thiophene, benzothiophene and thiophene as the coexisting compounds to prepare the model gasoline, and carried out adsorption experiments under the same conditions. Fig. 9 indicated that the equilibrium adsorption capacity was in the order of benzothiophene > thiophene $>3$-methyl thiophene. The adsorption mechanism is regarded as a combined effect of many factors involving appropriate framework structure, suitable pore size and shape of adsorbent, and characteristic of adsorbate. The MIL-101(Cr) nanoparticles in the membrane can binds organic sulfur compounds through two types of adsorption modes: $\pi$ complexation and direct coordination (S-M) interaction. As a result, the interaction between MIL-101(Cr) and benzothiophene is stronger than that of thiophene. Meanwhile, the size of 3-methyl thiophene molecular is larger than that of thiophene, which led to an easier adsorbing of MIL-101(Cr) for thiophene than for 3-methyl thiophene.

\section{Conclusion}

In present study, a novel porous membrane adsorbent MIL101(Cr)/SA was prepared by loading MIL-101(Cr) nanoparticles into SA matrix and used for adsorption of thiophene from model gasoline. The experimental results show that the porous MIL-101(Cr)/SA hybrid membrane exhibited a superior uptake capacity of $671 \mathrm{mg} \mathrm{g}^{-1}$ main due to $\pi$-complexation and direct coordination (S-M) interaction of MIL-101(Cr) with thiophene. The kinetics adsorption data could be well fitted with pseudosecond-order kinetic model. Meanwhile, the kinetics data were tested by Crank diffusion model also showed that the adsorption of thiophene by porous MIL-101(Cr)/SA hybrid membrane was mainly controlled by the intraparticle diffusion. The successive adsorption-desorption experiment showed that the porous MIL-101(Cr)/SA hybrid membrane could be reused with excellent stability. Therefore, it can be believed that the prepared novel porous MIL-101(Cr)/SA hybrid membrane adsorbent is a promising absorbent for removing thiophene from gasoline with high efficiency.

\section{Conflicts of interest}

There are no conflicts to declare.

\section{Acknowledgements}

The authors would like to acknowledge the financial support of this work from National Natural Science Foundation of China (No. 21676133). The authors also thank the anonymous referees for comments on this manuscript.

\section{References}

1 Y. X. Yang, G. Q. Lv, J. Li, et al., J. Alloys Compd., 2018, 747, 189.

2 Y. X. Yang, G. Q. Lv, L. L. Deng, et al., J. Cleaner Prod., 2017, $161,422$.

3 Y. C. Shi, W. Zhang, H. X. Zhang, et al., Fuel Process. Technol., 2013, 110, 24.

4 F. Subhan, S. Aslam, Z. F. Yan, et al., Microporous Mesoporous Mater., 2014, 199, 108.

5 C. M. Meng, Y. M. Fang, L. J. Jin and H. Q. Hu, Catal. Today, 2010, 149, 138.

6 X. Liu, J. Y. Wang, Q. Y. Li, et al., J. Rare Earths, 2014, 32, 189.

7 J. A. Vallaa, A. A. Lappas, I. A. Vasalos, et al., Appl. Catal., A, 2004, 276, 75.

8 M. Toba, Y. Miki, Y. Kanda, et al., Catal. Today, 2005, 104, 64. 9 Z. Hasan, J. Jeon and S. H. Jhung, J. Hazard. Mater., 2012, 205-206, 216.

10 W. F. De Souza, I. R. Guimaraes, M. C. Guerreiro and L. C. A. Oliveira, Appl. Catal., A, 2009, 360, 205.

11 J. Zhang, A. Wang, Y. Wang, et al., Chem. Eng. J., 2014, 245, 65.

12 H. Song, J. Gao, X. Chen, et al., Appl. Catal., A, 2013, 456, 67. 13 F. Davoodi-Dehaghani, M. Vosoughi and A. A. Ziaee, Bioresour. Technol., 2010, 101, 1102.

14 A. Ates, G. Azimi, K. H. Choi, et al., Appl. Catal., B, 2014, 147, 144.

15 H. Song, X. Wan, M. Dai, et al., Fuel Process. Technol., 2013, 116, 52.

16 I. M. Valverde Jr, J. F. Paulino and J. C. Afonso, Quim. Nova, 2008, 31, 680.

17 Y. C. Qin, Z. S. Mo, W. G. Yu, et al., Appl. Surf. Sci., 2014, 292, 5.

18 L. Lin, Y. Zhang, H. Zhang and F. Lu, J. Colloid Interface Sci., 2011, 360, 753.

19 L. Duan, X. Gao, X. Meng, et al., J. Phys. Chem. C, 2012, 116, 25748.

20 Y. Shi, X. Yang, F. Tian, et al., J. Nat. Gas Chem., 2012, 21, 421.

21 T. k A. Saleh, K. O. Sulaiman, S. A. AL-Hammadi, et al., J. Cleaner Prod., 2017, 154, 401.

22 F. Subhan, B. S. Liu, Q. L. Zhang and W. S. Wang, J. Hazard. Mater., 2012, 239-240, 370.

23 R. M. Cavalcanti, W. A. G. Pessoa Júnior, V. S. Braga and I. d. C. L. Barros, Appl. Surf. Sci., 2015, 355, 171.

24 F. Habimana, D. Shi and S. F. Ji, Appl. Clay Sci., 2018, 152, 303.

25 L. J. Xue, D. Zhang, Y. Q. Xu and X. M. Liu, Microporous Mesoporous Mater., 2017, 238, 46.

26 M. Ye, Y. F. Zhang, L. D. Li, et al., Appl. Surf. Sci., 2015, 346, 165.

27 C. J. Li, Y. J. Li, J. N. Wang, et al., Chem. Eng. J., 2013, 222, 419.

28 A. J. Hernandez-Maldonado, S. D. Stamatis, R. T. Yang, et al., Ind. Eng. Chem. Res., 2004, 43, 769.

29 H. C. Zhou, J. R. Long and O. M. Yaghi, Chem. Rev., 2012, 112, 673. 
30 F. P. Tian, Q. F. Ru, C. X. Qiao, et al., J. Energy Chem., 2018, 001, 1.

31 K. Yang, Y. Yan, W. Chen, et al., Polyhedron, 2018, 152, 202. 32 Z. P. Zhao, Z. Zuhra, L. B. Qin, et al., Fuel Process. Technol., 2018, 176, 276.

33 M. M. Nasef and A. H. Yahaya, Desalination, 2009, 249, 677.

34 J. H. Chen, X. Sun, W. Weng, et al., Chem. Eng. J., 2015, 273, 19.

35 L. L. Min, Z. H. Yuan, L. B. Zhong, et al., Chem. Eng. J., 2015, 267, 132.
36 M. M. Lakouraj, F. Mojerlou and E. N. Zare, Carbohydr. Polym., 2014, 106, 34.

37 J. H. Chen, X. Sun, L. J. Lin, et al., Chinese J. Chem. Eng., 2017, 25, 775 .

38 Z. Hasan, J. W. Jun and S. H. Jhung, Chem. Eng. J., 2015, 278, 265.

39 Y. T. Xia, Y. K. Li, Y. T. Gu, et al., Fuel, 2016, 170, 100.

40 J. H. Chen, J. C. Ni, Q. L. Liu and S. X. Li, Desalination, 2012, 285, 54.

41 C. O. Illanes, N. A. Ochoa and J. Marchese, Chem. Eng. J., 2008, 136, 92. 